\title{
Lipid Abnormalities in the Natural History of Diabetes
}

\author{
MUHAMMAD HAFIZUR RAHMAN, ${ }^{1}$ LIAQUAT ALI ${ }^{2}$
}

\begin{abstract}
:
Objective: To explore lipid abnormalities in normoglycemic first-degree diabetic relatives (FDRs) and prediabetic and diabetic subjects in the natural history of diabetes.

Research design and methods: Thirty six impaired fasting glucose (IFG), 61 isolated impaired glucose tolerance (I-IGT), 64 combined IFG-IGT, 73 diabetic, and 32 FDRs along with 57 normoglycemic healthy controls without family history of diabetes in It degree relatives, were selected purposively following 2003 ADA cut-off values and 2006 WHO/IDF grouping. Anthropometry and blood pressure of the subjects were taken. Fasting and 2-h plasma glucose and $\mathrm{HbA}_{I C}$ were measured. Fasting plasma triglyceride, total cholesterol and HDL cholesterol were measured by enzymatic colorimetric method.

Results: Serum triglyceride was higher in IFG, I-IGT, IFG-IGT, diabetic and FDRs compared to Control [ 145 (59307), I 28 (66-584), I 66 (68-764), I 6 I (69-750) and I 30 (8I-28I) vs. 108 (47-2l9) mg/dl, $P<0.0 I$, $P<0.0 I$, $P<0.00 I, P<0.001$ and $P<0.05]$. Total cholesterol was raised in IFG-IGT and diabetes compared to Control [ 185 (I05-3I0), I85 (I23-326) vs. I 7 I (I0I-235) mg/dl, P<0.05] and FDRs. But HDL did not differ among the groups. Prevalence of metabolic syndrome was higher in IFG, I-IGT, IFG-IGT and diabetes and FDRs than Control [55\%, 38\%, 57\%, 58\% and 36\% vs. $15 \%, P<0.00 I, P<0.01, P<0.00 I, P<0.00 I$ and $P<0.05]$ and also in IFGIGT and diabetic compared to I-IGT and FDRs $(P<0.05)$.

Conclusions: Higher prevalence of metabolic syndrome and raised serum triglyceride is seen among diabetic, prediabetic and I ${ }^{\text {st }}$ degree diabetic-relatives. Total cholesterol and non-HDL cholesterol is raised only in IFG-IGT and diabetes, the more decompensated glycemic states.
\end{abstract}

Key words: IFG, IGT, combined IFG-IGT, normoglycemic first-degree diabetic relatives, serum triglyceride, metabolic syndrome.

\section{Introduction}

Individuals in the natural history of type 2 diabetes pass through different stages. ${ }^{1}$ The first stage begins at birth, when glucose homeostasis is normal but individuals are at risk for developing diabetes because of inherited non-specific diabetogenic genes or a compromised intrauterine environment predisposing them to limit the ability of their pancreatic b cells to compensate for insulin resistance. In the next stage, insulin resistance emerge, probably due to interaction of environmental factors with genetic factors, which are initially compensated for by an increase in b-cell function, so that glycemic profile is normal even with glucose challange. The next stage is prediabetic stage, collectively termed as 'prediabetes' or 'categories of increased risk for diabetes' by ADA Expert Committee ${ }^{2,3}$ and 'Intermediate

1. Associate Professor, Department of Endocrinology, Dhaka Medical College and Hospital, Dhaka-1000, Bangladesh.

2. Professor, Department of Biochemistry and Cell Biology, and Director, BIHS; Coordinator, Biomedical Research Group, BIRDEM, Dhaka, Bangladesh.

Correspondence: Dr Muhammad Hafizur Rahman, Associate professor, Department of Endocrinology, Dhaka Medical College and Hospital, Dhaka-1000, Bangladesh. Tel.: 880-1199063141. E-mail: hafizdrendo@yahoo.com
Hyperglycemia’ by WHO/IDF Technical Advisory Group. ${ }^{4}$ In this stage b-cell function and insulin sensitivity both deteriorate, so both challenged (with glucose or meal) and basal blood glucose is raised above the normal range but below the diagnostic cut point for diabetes. When fasting plasma glucose (FPG) is raised it is termed as 'impaired fasting glucose' (IFG), and when 2-h plasma glucose (2-h PG) value in the oral glucose tolerance test is raised it is termed as 'isolated impaired glucose tolerance' (I-IGT). When both fasting and 2-h value is raised it is termed as combined IFG-IGT. Ultimately, as a result of further deterioration in b-cell function, subjects enter into diabetic stage when fasting and/or postprandial glucose levels reach diabetic range.

Diabetes mellitus is associated with multiple abnormalities in lipid metabolism but the underlying mechanisms are complex. Type 2 diabetic subjects show mild hypertriglyceridemia accompanied by reduced HDL cholesterol. ${ }^{5-7}$ LDL cholesterol is normal or only slightly raised, but total cholesterol is similar to general population. ${ }^{5,6}$ Diabetic hypertriglyceridemia results from excessive production of VLDLs by the liver, as well as reduced clearence of TG-rich lipoproteins due to decreased activity 
of LPL enzyme. ${ }^{5,8}$ The prolonged residence time of the TGrich lipoproteins in the circulation leads to increased exchange of their TG for cholesteryl esters in HDLs as well as in LDLs by the cholesteryl ester transfer protein (CETP). This increased bidirectional TG-cholesteryl ester exchange produce TG-enriched HDLs and LDLs, which are then hydrolyzed by hepatic lipase to produce small dense LDL and HDL particles; the latter are rapidly cleared from circulation, leading to lower serum HDL cholesterol. ${ }^{5}$

There are very few studies in prediabetic subjects and in normoglycemic $1^{\text {st }}$ degree diabetic-relatives (FDRs) regarding lipid abnormalities. Studies in FDRs has found raised $\mathrm{TG}^{9,10}$ and decreased HDL. ${ }^{11}$ But there are very limited studies to find lipid abnormalities in each prediabetic groups. High level of TG was found in IFG, IGT, IFG-IGT, ${ }^{12-}$ 16 and low HDL in IFG and IGT. ${ }^{13-15}$ Probably insulin resistance and/or secretion defect in these subjects have role for this dyslipidemia. There are also genetic factors and ethnic influence on lipid abnormalities. The present study was undertaken to explore lipid abnormalities in Bangladeshi prediabetic and diabetic subjects and FDRs.

\section{Research design and methods}

All newly detected prediabetic and diabetic subjects alone with FDRs were selected from the out-patient department of BIRDEM (the tertiary hospital of Bangladesh Diabetic Somity) every $3^{\text {rd }}$ working days during the period of January to June, 2005. Following the cut-off values of 2003 ADA Follow-up Report ${ }^{2}$ and grouping of 2006 WHO/IDF Consultation Report, ${ }^{4} 36$ IFG (FPG 5.6 - 6.9 mmol/l and 2h PG $<7.8 \mathrm{mmol} / \mathrm{l}$ ), 61 I-IGT (FPG $<5.6 \mathrm{mmol} / \mathrm{l}$ and 2-h PG 7.8 - $11.0 \mathrm{mmol} / \mathrm{l}), 64$ IFG-IGT (FPG $5.6-6.9 \mathrm{mmol} / \mathrm{l}$ and 2-h PG 7.8 - $11.0 \mathrm{mmol} / \mathrm{l}$ ), 73 diabetic subjects (FPG >6.9 $\mathrm{mmol} / \mathrm{l}$ and 2-h PG >11.0 mmol/l), and 32 FDRs along with 57 normoglycemic healthy controls with no family history of diabetes in first degree relatives (FPG $<5.6 \mathrm{mmol} / \mathrm{l}$ and 2-h PG $<7.8 \mathrm{mmol} / \mathrm{l}$ ), were selected. Patients with serious comorbid diseases or using drugs significantly affecting glucose metabolism (like glucocorticoids, oral contraceptives containing levonorgestrel or high-dose estrogen, phenytoin, high-dose thiazide diuretics etc.) or lipid metabolism (like lipid lowering drugs, beta blockers, diuretics, hormones etc.) or history of gestational diabetes mellitus and pregnant women were excluded. The purpose of the study was explained in details to each subject and informed written consent was taken. The study protocol was approved by the Ethical Committee of the Association.

\section{Anthropometric Measurements}

Height, weight, waist circumference (WC), neck circumference (NC) and blood pressure were measured using standard procedure. Then BMI of the subjects were calculated as weight/height ${ }^{2}\left(\mathrm{~kg} / \mathrm{m}^{2}\right)$. Subjects with higher diastolic and/or systolic blood pressure or under treatment with hypotensive drugs were considered hypertensive. Body fat mass was measured by Omron Body Fat Monitor.

\section{Oral glucose tolerance test}

After at least 3 days of unrestricted carbohydrate diet, avoiding strenuous exercise and fasting for 8-14 hours, the subjects underwent 75-g 2-h OGTT following WHO Guidelines. ${ }^{17}$ Blood was collected at fasting between 8.009.00 AM and then 2-h after 75-g glucose load.

\section{Laboratory data, analytical procedure and calculations}

Fasting and 2-h plasma glucose was measured by Glucose Oxidase method (Randox Laboratories Ltd., Co. Antrim, UK) and $\mathrm{HbA}_{1 \mathrm{C}}$ by VARIANT Hemoglobin $\mathrm{A}_{1 \mathrm{C}}$ Program (BioRad Laboratories, CA, USA) on the same day. Fasting plasma TG, TC and HDL cholesterol were measured by enzymatic colorimetric method (Randox Laboratories Ltd., UK).

Non-HDL cholesterol (=TC-HDL cholesterol), ratio of total cholesterol to HDL cholesterol (TC/HDL) and ratio of TG to HDL cholesterol (TG/HDL), markers of cardiovascular risk, were determined. Metabolic Syndrome (MetS), a cluster of risk factors for coronary artery disease, was defined using the IDF Consensus Worldwide definition of metabolic syndrome in 2006. ${ }^{18}$ The prevalence of MetS and its components were evaluated.

\section{Statistical analysis}

Statistical analysis was performed using SPSS software for Windows version 11.0 (SPSS Inc., Chicago, IL, USA). Sex, high WC, arterial blood pressure and TG, low HDL and MetS distribution were expressed as percent and compared in different groups by Chi Squared $\left(\chi^{2}\right)$ test. Age, BMI, NC and BFM were expressed as mean $\pm \mathrm{SD}$ and the statistical difference between the groups were assessed by One-Way ANOVA with Post-Hoc Benferroni. WC, fasting and $2 \mathrm{~h}$ plasma glucose, HbA1c, TG, TC, HDL, non-HDL cholesterol, TC/HDL and TG/HDL were not normally distributed and were expressed as median (range) and analysis for statistical difference were done by MannWhitney $U$ test. $P$ values less than 0.05 were considered significant.

\section{Results}

Clinical and glycemic status of the study subjects are shown in Table I. Diabetic subjects were a bit older than Control subjects. There were more female subjects in I-IGT group than Control, IFG, IFG-IGT and diabetic groups and also in 
FDRs than Control, IFG-IGT and diabetic subjects. BMI was higher only in diabetic subjects, and waist circumference was higher in IFG, IFG-IGT and diabetic subjects compared to Control. Neck circumference was higher in IFG-IGT and diabetic subjects than I-IGT subjects and FDRs, but not compared to Control. $\mathrm{HbA}_{1 \mathrm{C}}$ was higher in IFG-IGT and diabetic subjects than Control and FDRs and also higher in diabetic subjects than I-IGT subjects.

Serum triglyceride was higher in IFG, I-IGT, IFG-IGT, diabetic and FDRs compared to Control [145(59-307), 128(66-584), 166(68-764), 161(69-750) and 130(81-281) vs. 108 (47-219) $\mathrm{mg} / \mathrm{dl}, P<0.01, P<0.01, P<0.001, P<0.001$ and $P<0.05$ ] (Table II). Triglyceride was also higher in IFGIGT and DM compared to I-IGT $(P<0.01)$ and FDRs $(P<0.05)$. Similarly TG/HDL ratio was higher in IFG, I-IGT, IFG-IGT, diabetic and FDRs compared to Control [4.2(1.410.4), 3.9(1.4-17.9), 4.8(1.7-25.1), 4.5(1.6-20.1) and 4.1(1.3-6.9) vs. 2.9(1.0-6.7), $P<0.01, P<0.001, P<0.001$, $P<0.001$ and $P<0.01]$. This ratio was also higher in IFGIGT and diabetic compared to I-IGT $(P<0.01$ and $P<0.05)$. Serum HDL value did not vary among the groups. Total cholesterol level was raised in IFG-IGT and diabetes compared to Control [185(105-310), 185(123-326) vs. 171(101-235) mg/dl, $P<0.05]$ and FDRs [185(105-310), 185(123-326) vs. $159(130-260) \mathrm{mg} / \mathrm{dl}, P<0.05]$. Non HDL cholesterol level was similarly raised in IFG-IGT and diabetes compared to Control [147(85-270), 148(76-284) vs. 131(66-198), mg/dl, $P<0.05]$ and FDRs [147(85-270), 148(76-284) vs. $128(88-236)$, mg/dl, $P<0.05]$. TC/HDL ratio was also raised in IFG-IGT and diabetes compared to Control [5.3(3.2-10.5), 4.6(2.2-7.9) vs. 4.6(2.2-7.9), $P<0.01$ and $P<0.05]$.

When the components of MetS were compared among the groups (Table III), it was found that prevalence of high waist circumference was more in I-IGT, IFG-IGT and diabetes compared to Control (59\%, 59\% and 61\% vs. 38\%, $P<0.05$, $P<0.05$ and $P<0.01)$. Hypertension prevalence was more in IFG-IGT and diabetes compared to Control (51\% and 60\% vs. $29 \%, P<0.05$ and $P<0.01$ ) and I-IGT (51\% and $60 \%$ vs. $33 \%, P<0.05)$. Among lipid profile, raised TG was more prevalent in IFG, I-IGT, IFG-IGT and diabetes compared to Control (47\%, 35\%, 56\% and 53\% vs. 19\%, $P<0.01, P<0.05$, $P<0.01$ and $P<0.01)$. High TG was also found in IFG-IGT compared to I-IGT and FDRs (56\% vs. 35\% and 33\%, $P<0.05)$ and in diabetes compared to I-IGT $(P<0.05)$.Low HDL was found in I-IGT and FDRs compared to Control (90\% and 90\% vs. 70\%, $P<0.05$ ) and DM (90\% and 90\% vs. $74 \%, P<0.05)$. Prevalence of metabolic syndrome was higher in IFG, I-IGT, IFG-IGT, diabetes and FDRs than Control [55\%, 38\%, 57\%, 58\% and 36\% vs. 15\%, $P<0.001$, $P<0.01, P<0.001, P<0.001$ and $P<0.05]$ and also high in IFG-IGT and diabetic subjects compared to I-IGT and FDRs $(P<0.05)$.

\section{Table-I}

Clinical and glycemic profile of the study subjects $(n=323)$

\begin{tabular}{|c|c|c|c|c|c|c|}
\hline & $\begin{array}{l}\text { Control } \\
(\mathrm{n}=57) \\
\end{array}$ & $\begin{array}{c}\text { IFG } \\
(\mathrm{n}=36)\end{array}$ & $\begin{array}{c}\text { I-IGT } \\
(\mathrm{n}=61)\end{array}$ & $\begin{array}{c}\text { IFG-IGT } \\
(\mathrm{n}=64)\end{array}$ & $\begin{array}{c}\mathrm{DM} \\
(\mathrm{n}=73)\end{array}$ & $\begin{array}{c}\text { FHO-DM } \\
(\mathrm{n}=32)\end{array}$ \\
\hline Female (\%) & $20(35 \%)$ & $18(50 \%)$ & $46(74 \%)^{* * * \dagger}$ & $31(48 \%)^{\ddagger \neq}$ & $29(39 \%)^{\ddagger \#}$ & $23(69 \%)^{* * \S}$ \\
\hline Age (yrs) & $39 \pm 11$ & $43 \pm 11$ & $40 \pm 11$ & $43 \pm 9$ & $45 \pm 10^{*}$ & $39 \pm 9$ \\
\hline $\mathrm{BMI}\left(\mathrm{kg} / \mathrm{m}^{2}\right)$ & $23.3 \pm 3.9$ & $25.2 \pm 3.5$ & $24.8 \pm 4.2$ & $25.4 \pm 3.9$ & $25.6 \pm 3.5^{*}$ & $24.9 \pm 3.5$ \\
\hline $\mathrm{WC}(\mathrm{cm})$ & 80(57-106) & $90(61-104){ }^{*}$ & $86(59-108)$ & $90(59-110)^{* * \ddagger}$ & $90(61-110)^{* * \neq \neq}$ & $85(58-98)^{\S}$ \\
\hline $\mathrm{NC}(\mathrm{cm})$ & $34 \pm 3$ & $35 \pm 3$ & $33 \pm 3$ & $36 \pm 3^{\ddagger \neq}$ & $35 \pm 3^{\ddagger \neq}$ & $33 \pm 3^{\S}$ ๆ \\
\hline BFM (\%) & $27.9 \pm 7.2$ & $31.0 \pm 6.0$ & $31.0 \pm 6.2$ & $30.3 \pm 6.6$ & $30.6 \pm 6.2$ & $30.3 \pm 7.0$ \\
\hline F-Glu (mmol/l) & $4.9(2.8-5.5)$ & $5.9(5.6-6.9)^{* * *}$ & $5.0(4.1-5.5)^{\dagger+\dagger}$ & $\begin{array}{c}5.9(5.6-6.9) \\
* * * \neq \neq \ddagger\end{array}$ & $\begin{array}{c}6.3(3.6-10.0) \\
* * * \dagger \neq \# \ddagger \S \S\end{array}$ & $\begin{array}{c}5.0(3.8-5.5) \\
+\dagger † \S \S \S \text { ศศ }\end{array}$ \\
\hline 2-h-Glu (mmol/l) & $6.0(3.4-7.6)$ & $6.8(4.2-7.7)^{* *}$ & $\begin{array}{c}9.0(7.8-11.0) \\
* * *+\dagger \dagger\end{array}$ & $\begin{array}{c}9.7(7.9-11.0) \\
* * *+\dagger \dagger \neq\end{array}$ & $\begin{array}{c}12.3(8.3-18.7) \\
* * *+\dagger \dagger \neq \# \neq\end{array}$ & 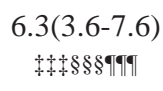 \\
\hline $\mathrm{HbA}_{1 \mathrm{C}}(\%)$ & $5.6(4.5-6.5)$ & $5.5(4.7-7.1)$ & $5.7(4.3-7.9)$ & $5.8(4.3-7.3)^{* *}$ & $\begin{array}{c}5.9(4.8-8.2) \\
* * \neq\end{array}$ & $\begin{array}{c}5.4(4.7-6.1) \\
\text { §ษศศศ }\end{array}$ \\
\hline
\end{tabular}

Data are $\mathrm{n}(\%)$, mean \pm SD or median (range). $* P<0.05$, ** $P<0.01$ and $* * * P<0.001$ vs. Control, $\dagger P<0.05$ and $\dagger \dagger \dagger P<0.001$ vs. IFG, $\ddagger$

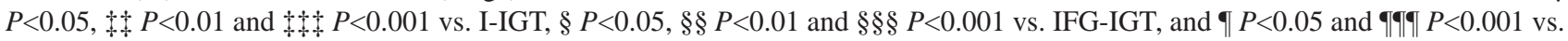
DM. $n$ = number of subjects. BMI, body mass index; WC, waist circumference; NC, neck circumference; BFM, body fat mass; F, fasting; 2-h, 2 hour after 75-g glucose, Glu, glucose. 
Table II

Lipid profile of the study subjects

\begin{tabular}{|c|c|c|c|c|c|c|}
\hline & Control & IFG & I-IGT & IFG-IGT & DM & FHO-DM \\
\hline $\mathrm{TG}(\mathrm{mg} / \mathrm{dl})$ & $108(47-219)$ & $145(59-307)^{* *}$ & $128(66-584)^{* *}$ & $166(68-764)^{* * * \neq \ddagger}$ & $161(69-750)^{* * * \neq \ddagger}$ & $130(81-281) * \$$ \\
\hline HDL(mg/dl) & $38(24-62)$ & $36(16-53)$ & $35(22-59)$ & $35(19-49)$ & $38(20-58)$ & $34(23-54)$ \\
\hline $\mathrm{TC}(\mathrm{mg} / \mathrm{dl})$ & $171(101-235)$ & $180(102-248)$ & 180(102-286) & $185(105-310)^{*}$ & $185(123-326)^{*}$ & $159(130-260)$ \\
\hline Non HDL(mg/dl) & 131(66-198) & $142(69-212)$ & 146(73-250) & $147(85-270)^{*}$ & $148(76-284)^{*}$ & $128(88-236))^{8 \uparrow}$ \\
\hline TC/HDL(mg/dl) & 4.6(2.2-7.9) & $5.0(3.1-9.6)$ & $5.0(2.9-8.8)$ & $5.3(3.2-10.5)^{* *}$ & $4.6(2.2-7.9)^{*}$ & $5.0(2.6-10.4)$ \\
\hline TG/HDL(mg/dl) & $2.9(1.0-6.7)$ & $4.2(1.4-10.4)^{* *}$ & $3.9(1.4-17.9)^{* * *}$ & $4.8(1.7-25.1)^{* * * \neq \ddagger}$ & $4.5(1.6-20.1)^{* * * \ddagger}$ & $4.1(1.3-6.9)^{* *}$ \\
\hline
\end{tabular}

Data are median (range). $* P<0.05, * * P<0.01$ and $* * * P<0.001$ vs. Control, $\ddagger P<0.05$ and $\neq \ddagger \neq P<0.01$ vs. I-IGT, § $P<0.05$ vs. IFG-IGT, and $\uparrow<0.05$ vs. DM. TG, triglyceride: TC, total cholesterol.

Table III

Frequency of abnormal cardiometabolic variables of the study subjects

\begin{tabular}{|c|c|c|c|c|c|c|}
\hline & Control & IFG & I-IGT & IFG-IGT & $\mathrm{DM}$ & FHO-DM \\
\hline High WC & $22(38 \%)$ & $21(58 \%)$ & $37(59 \%)^{*}$ & $38(59 \%)^{*}$ & $45(61 \%)^{* *}$ & $18(54 \%)$ \\
\hline \multicolumn{2}{|c|}{ Arterial Hypertension17 (29\%) } & 15 (41\%) & $21(33 \%)$ & $33(51 \%)^{* \ddagger}$ & $44(60 \%)^{* * \ddagger}$ & $11(33 \%)^{\pi}$ \\
\hline High TG & $11(19 \%)$ & $17(47 \%)^{* *}$ & $22(35 \%)^{*}$ & $36(56 \%)^{* * * \ddagger}$ & $39(53 \%)^{* * * \ddagger}$ & $11(33 \%)^{\S}$ \\
\hline Low HDL & $40(70 \%)$ & $30(83 \%)$ & $56(90 \%)^{*}$ & $53(82 \%)$ & $54(74 \%)^{\ddagger}$ & $30(90 \%)^{*}$ \\
\hline High F-Glu & $0(0 \%)$ & $36100 \%)^{* * *}$ & $0(0 \%)^{\dagger+\dagger}$ & $64(100 \%)^{* * * *+\neq}$ & $73(100 \%)^{* * *+\neq ⿰ 木 \neq}$ & $0(0 \%)^{\dagger \dagger \dagger \S \S \S \uparrow ศ ศ 币}$ \\
\hline MetS & 9 (15\%) & $20(55 \%)^{* * *}$ & $24(38 \%)^{* *}$ & $37(57 \%)^{* * * \ddagger}$ & $43(58 \%)^{* * * \ddagger}$ & $12(36 \%)^{* \S \Phi}$ \\
\hline
\end{tabular}

Data are $\mathrm{n}(\%) . * P<0.05, * * P<0.01$ and $* * * P<0.001$ vs. Control, $\dagger \dagger \dagger P<0.001$ vs. IFG, $\ddagger P<0.05$ and $\neq \neq \neq+P<0.001$ vs. I-IGT, § $P<0.05$, and $\S \S \S P<0.001$ vs. IFG-IGT, and $\uparrow P<0.05$ and $\uparrow \uparrow \uparrow \uparrow<0.001$ vs. DM. WC, waist circumference; TG, triglyceride; F, fasting; Glu, glucose; MetS, metabolic syndrome.

\section{Conclusions}

Our study finding shows higher TG in IFG, I-IGT, IFG-IGT, diabetes and FDRs (Table II). Hypertriglyceridemia is a feature of type 2 diabetes. ${ }^{5,8}$ Higher TG is also a feature of prediabetic subjects. ${ }^{12-16}$ Insulin resistance and/or B-cell failure in prediabetic and diabetic subjects may be responsible for higher TG in these subjects. Studied in FDRs have shown raised $\mathrm{TG}^{9,10}$ and insulin resistance. ${ }^{10,19}$ Higher TG in FDRs may be due to insulin resistance. Our study finding also shows raised TG in IFG-IGT and diabetes compared to I-IGT and FDRs. As $\mathrm{HbA}_{1 \mathrm{C}}$ levels are higher in IFG-IGT and diabetes than Control (Table I), I-IGT and FDRs, IFG-IGT and diabetes are more decompensated glycemic states, which may explain higher TG in these subjects.

HDL level does not differ among the groups. But TG/HDL ratio is a better marker of dyslipidemia, which, like TG, is higher in prediabetic and diabetic subjects and FDRs and also higher in IFG-IGT and diabetic subjects than I-IGT subjects.
In the study, we have also found that prevalence of MetS is higher in prediabetic and diabetic subjects and FDRs (Table III). It is well accepted that MetS is associated with insulin resistance. So insulin resistance in these subjects may be responsible for higher prevalence of MetS in them and also explain raised TG in these subjects.

Total cholesterol and non-HDL cholesterol is higher in IFGIGT and diabetic subjects than control and FDRs. TC/HDL ratio is also raised in IFG-IGT and diabetes than Control. As mentioned before, IFG-IGT and diabetes are more decompensated glycemic states. So total cholesterol, nonHDL cholesterol and TC/HDL ratio is raised only in more decompensated glycemic states of IFG-IGT and diabetes.

In our study, FDRs have raised TG and TG/HDL ratio, even though blood glucose level is within normal range. So raised TG may be the earliest metabolic change in FDRs. Prediabetic subjects (IFG, I-IGT and IFG-IGT) have raised blood glucose but within prediabetic range. Among the measured parameters of lipid profile, only TG is raised in 
all prediabetic subgroups. So raised TG is the earliest lipid abnormality in prediabetic subjects. Among the prediabetic subgroups, only IFG-IGT has raised total cholesterol, nonHDL cholesterol and TC/HDL ratio, which is also seen in diabetic subjects. As IFG-IGT and diabetes are more decompensated glycemic states, total cholesterol and nonHDL cholesterol is raised only in more decompensated glycemic states, viz. IFG-IGT and diabetes.

Using cut off values of the IDF Consensus Worldwide definition of metabolic syndrome, ${ }^{18}$ high WC is more prevalent in I-IGT, IFG-IGT and diabetes, and hypertension is more prevalent in IFG-IGT and diabetes. In lipid profile, high TG is prevalent in prediabetic and diabetic subjects and FDRs and low HDL cholesterol in I-IGT and FDRs. High fasting plasma glucose is prevalent in IFG, IFG-IGT and diabetes, as defined in case selection. When MetS is defined from its components, it is found that prevalence of MetS is higher in prediabetic and diabetic subjects and FDRs.

Although absolute value of HDL cholesterol did not vary among the groups, but when subjects were grouped as having low and high HDL cholesterol, it was evident that low HDL cholesterol was more prevalent in I-IGT and FDRs. We know that FDRs suffer insulin resistance. ${ }^{19}$ In a Bangladeshi population, it was found that IFG subjects have B-cell dysfunction, I-IGT subjects suffer insulin resistance, and IFG-IGT subjects suffer both Bcell dysfunction and insulin resistance. ${ }^{20}$ It is well known that type 2 diabetic patients suffer both B-cell failure and insulin resistance. So low HDL cholesterol is prevalent only in those Bangladeshi subjects which have pure insulin resistance and not accompanied by B-cell failure.

So, in conclusion, it is evident from the study that raised serum triglyceride level is the earliest metabolic derangement in $1^{\text {st }}$ degree diabetic-relatives and the earliest lipid abnormality in prediabetic subjects. Total cholesterol and non-HDL cholesterol is raised in IFG-IGT and diabetes, the more decompensated glycemic states. Higher prevalence of metabolic syndrome is seen among diabetic, prediabetic and $1^{\text {st }}$ degree diabetic-relatives.

\section{Acknowledgements}

We gratefully acknowledge the financial supports of International Program in the Chemical Sciences (IPICS), Uppsala University, Sweden and Diabetic Association of Bangladesh (DAB) in conducting the study.

\section{Conflict of Interest: None}

\section{References}

1. Gerich JE, Smith TM. b-Cell defects and pancreatic abnormalities in type 2 diabetes. In: Textbook of Diabetes, 3rd ed. John C. Pickup \& Gareth Williams, Eds, Blackwell Science, 2003:23.1-23.11
2. The Expert Committee on the Diagnosis and Classification of Diabetes Mellitus. Follow up Report on the Diagnosis of Diabetes Mellitus. Diabetes Care 2003;26:3160-3167.

3. American Diabetic Association. Diagnosis and Classification of Diabetes Mellitus. Diabetes Care 2003;33(1):62-69.

4. World Health Organization and International Diabetes Federation. Definition and Diagnosis of Diabetes Mellitus and Intermediate Hyperglycemia. Report of a WHO/IDF Consultation. Geneva: World Health Organization, 2006.

5. Poirier P, Despres JP. Lipid disorders in diabetes. In: Textbook of Diabetes, 3rd ed. John C. Pickup \& Gareth Williams, Eds. Massachusetts, Blackwell Science, 2003:54.1-54.21.

6. Howard BV. Lipoprotein metabolism in diabetes mellitus. J Lipid Res 1987;12:613-28.

7. Steiner G. Dyslipoproteinemias in diabetes. Clin Invest Med 1995;18:282-7.

8. Ginsberg HN. Lipoprotein physiology in nondiabetic and diabetic states: relationship to atherogenesis. Diabetes Care 1991;14:839-55.

9. Florez H, Ryder E, Campos G et al. Women relatives of Hispanic patients with type 2 diabetes are more prone to exhibit metabolic disturbances. Invest Clin 1999;40(2): 127-42.

10. Laws A, Stefanick ML, Reaven GM. Insulin resistance and hypertriglyceridemia in nondiabetic relatives of patients with noninsulin-dependent diabetes mellitus. J Clin Endocrinol Metab 1989;69(2):343-7.

11. Shaw JT, Purdie DM, Neil HA, Levy JC, Turner RC. The relative risks of hyperglycaemia, obesity and dyslipidaemia in the relatives of patients with Type II diabetes mellitus. Diabetologia 1999;42(1):24-7.

12. Amoah AG, Schuster DP, Gaillard T, Osei K. Insulin resistance, beta cell function and cardiovascular risk factors in Ghanaians with varying degrees of glucose tolerance. Ethn Dis 2002;12(4):3-7.

13. Unwin W. The prevention or delay of type 2 diabetes. Diabetic Medicine 2002;19:708-723.

14. Burchfiel CM, Hamman RF, Marshall JA, Baxter J, Kahn LB, Amirani JJ. Cardiovascular risk factors and impaired glucose tolerance: the San Luis Valley Diabetes Study. Am J Epidemiol 1990;131(1):57-70.

15. Salomaa VV, Tuomilehto J, Jauhiainen M, Korhonen HJ, Stengård J, Uusitupa M, Pitkanen M, Penttila I. Hypertriglyceridemia in different degrees of glucose intolerance in a Finnish population-based study. Diabetes Care 1992;15(5):657-65. 
16. Novoa FJ, Boronat M, Saavedra P, Diaz-Cremades JM, Varillas VF, La Roche F, Alberiche MP, Carrillo A. Differences in cardiovascular risk factors, insulin resistance, and insulin resistance in individuals with normal glucose tolerance and in subjects with impaired glucose regulation: the Telda Study. Diabetes Care 2005;28:2388-2393.

17. World Health Organization. Definition, Diagnosis and Classification of Diabetes Mellitus and its Complications, Part 1: Diagnosis and Classification of Diabetes Mellitus, Report of a WHO Consultation, World Health Organization, Geneva, 1999.
18. Alberti KG, Zimmet P, Shaw J. Metabolic syndrome-a new world-wide definition. A Consensus Statement from the International Diabetes Federation. Diabet Med 2006;23(5): 469-80.

19. Henriksen JE, Alford F, Handberg A, Vaag A, Ward GM, Kalfas A, Beck-Nielsen H. Increased glucose effectiveness in normoglycemic but insulin-resistant relatives of patients with non-insulin-dependent diabetes mellitus. A novel compensatory mechanism. J Clin Invest 1994;94(3): 1196-1204.

20. Rahman MH, Hafizur RM, Nahar Q, Khan AR, Ali L. Insulin secretion and sensitivity in Bangladeshi prediabetic subjects. J Diabetes Complications 2010;24(1):37-42. 